\title{
Enhancement of psychomotor skills in children with autism spectrum disorder by employing a mechatronic training kit
}

https://doi.org/10.1515/pjbr-2019-0001

Received February 7, 2018; accepted October 1, 2018

\begin{abstract}
Prevalence of Autism Spectrum Disorder (ASD) in children has been increasing over the years. These children, when compared to typically growing children, face challenges in leading a day-to-day life. Other than the social interactions, communication and cognitive skills, psychomotor skill deficits are also found in them. This paper focuses on enhancement of psychomotor skills in children with ASD by employing a mechatronic training kit. Training using this kit aims at developing skills like 'palmar grasp', 'wrist rotation' and 'eye-hand coordination'. Trials were conducted with six children with autism aged between four and nine years. Significant improvements were seen in these children both in skills and in behaviour after the training. Thus, teaching psychomotor skills to children with autism using the newly developed mechatronic door training kit is found to be working and the results are encouraging.
\end{abstract}

Keywords: psychomotor skills, palmar grasp, wrist rotation, eye-hand coordination, mechatronic door training kit

\section{Introduction}

Prevalence of Autism Spectrum Disorder (ASD) in children has been increasing over the years. As per United States

\footnotetext{
Ramya S. Moorthy: School of Mechanical Engineering, SASTRA Deemed to be University, Thanjavur-613 401, Tamil Nadu, India; E-mail: ramyasmoorthy@gmail.com

Kritika Iyer: School of Mechanical Engineering, SASTRA Deemed to be University, Thanjavur-613 401, Tamil Nadu, India; E-mail:

kritikaiyer811@gmail.com

R. Hari Krishnan: School of Mechanical Engineering, SASTRA Deemed to be University, Thanjavur-613 401, Tamil Nadu, India; E-mail: harikrishnan.r@mech.sastra.edu

^Corresponding Author: S. Pugazhenthi: School of Mechanical Engineering, SASTRA Deemed to be University, Thanjavur-613 401, Tamil Nadu, India; E-mail: pugazhenthi@mech.sastra.edu
}

Centers for Disease Control and Prevention (CDC) report published in 2014, 1 in 68 American children come under the umbrella of ASD [1]. The recent report of CDC published in 2018 shows the change in figure to be 1 in 59, with an increase of $15 \%$ from the previous statistics [2]. Children with ASD often find it difficult to interact or communicate socially with outer world, when compared to typically growing children and this may lead to difficulties in their later life. They may have hindrances in making eye contact and some may have added issues such as epilepsy, gastro intestinal problems, and other neurological disorders [3]. A few of them may even have difficulties in helping themselves to use bathroom or for performing any of the work that is done easily by the rest of the population. Almost $80 \%-90 \%$ of the children with ASD are reported to have motor skill problems as well [4].

Weak motor skills are a universal problem in most children under the autistic spectrum [5]. Motor skill deficits are one of the contributing factors to dyspraxia in children with ASD [6]. In a research work involving 101 children with ASD, $79 \%$ of the children were found to have severe movement impairments and $10 \%$ of them had borderline problems [7].The cerebellum and the frontal lobe of the cerebrum are responsible for motor skill development. These areas are affected and hence the children cannot perform tasks with the coordination that is expected $[8,9]$. Hand to eye coordination is a difficult skill that makes the simplest of tasks seem like herculean tasks. Crippa et al. [10] investigated eye-hand coordination in children with ASD by comparing them with typically developing children. It was found that children with ASD showed less efficient eye-hand coordination than typically developing children. It has also been observed that children with ASD do not have bi-lateral coordination, which indicates the need for improvement in using both hands [11]. By making the children perform day-to-day tasks repeatedly as an exercise, it is possible to forge new neural pathways and help them function better [12]. In a study done at The Washington University, the motor impairments in children with ASD and their typically developing siblings were eval- 
uated. During the evaluation, the children were asked to place pegs in pegboard, cutting with scissors, throwing a ball, etc. The researchers used a standardized tool for evaluation to measure fine manual control, manual \& body coordination and strength \& agility. It was found that $83 \%$ of the children with ASD had a severe deficit in the motor skills and also found that there is a link between motor skills deficit and severity of ASD [13]. Evidence showing deficit of gross \& fine motor skills, lack of postural control and praxis impairments in infants were also reported [14]. Impaired motor imitation is observed as early as 20 months of age in children who are later diagnosed with autism $[15,16]$. Bremer has suggested that, by the intervention for Fundamental Motor Skills (FMS), which was ignored by previous researchers, improvements in motor skills and individual behaviour were seen [17]. Jasmine et al. proved that sensorimotor skills are highly correlated to Daily Life Skills (DLS) and also suggested that interventions have to be developed to support and address these skills which in turn will help in enhancing DLS [18]. Hence, developing motor skills is an extremely important part of intervention of ASD because it teaches the child to work on its own with minimum support and guidance by others. This paper focuses on enhancement of psychomotor skills in children with ASD by employing a mechatronic training kit.

Conventionally, occupational therapy and speech therapy are used as rehabilitative interventions [19]. Occupational therapy is a holistic approach which considers the physical, social, emotional, sensory and cognitive abilities of the children. It is done in various settings like school, home, play, etc. In all these setups, the activities that are addressed include social stories, social skills lessons, jump starters, social skills activities for special needs, etc. The children are also taken to field trips, sports events and playgrounds to enhance social interactions. During these therapy sessions, the children are asked to indulge in some activities, play or exercise their arm muscles to improve motor sensory skills. Though these are effective to some extent, there are limitations. The children find it difficult to communicate with the instructors due to lack of communication skills and unwillingness to make eye contact [20]. Moreover, the children do not find the activities interesting as they do not have any involvement or active role in performing the same. With robotic systems, the children break the barrier for communication and seem to feel more comfortable around them. These robotic systems are in attractive colours, shapes and sizes so that the children are drawn to it. Robots are not to replace therapists; they are used to assist therapists as there will always be a need for a human touch. Researchers have studied the positive effect that robotic systems have on ASD affected children; they show increased levels of motivation and engagement to the tasks as compared to those without the robots [21]. Studies using touch ball shows that the Human Robot Interaction (HRI) increases when the robot gives a positive feedback to the user [22].

Various projects and research programs have made use of robotic intervention to improve the quality of life of individuals with ASD. A study conducted by Goldsmith \& LeBlanc in 2004 has suggested that technological interventions including robotics will be of great use in helping children with ASD[23]. In 2003, Billard fabricated a robot, 'Robota', a humanoid doll to aid typically developing \& cognitively impaired children and children with autism through imitation [24]. The doll was loaded with simple inputs for language and was provided with artificial intelligence features. The children imitate what 'Robota' does and this in turn trains them in speech processing and social interaction at undergraduate levels of the curriculum. Kronreifin his research had suggested two projects, one using 'PlayROB', a remote controlled device to assist special children to play with and the other using 'Interactive Robotic social Mediators as Companions (IROMEC)'- a device on which children can draw, write, or trace their movements and interact [25]. The author suggests that toys realized with latest technologies should be accessible to children with disabilities in order to train them along with playing. Ranatunga et al. used Kinect sensors connected to humanoid robot 'Zeno' to mimic the therapist's movement and verify if the child was performing the same [26]. This was used to improve the motor skills of children with ASD, by performing tasks without social complication. Making use of a customized pick and place robot has been reported to be effective to impart concept of directions (up, down, right and left), palmar grasp and eye-hand coordination [27].

Stone et al. suggested that imitation of a certain act by children would help improve their skills; the robot performs the act and the children imitate the same [28]. They compared various motor imitations in 18 children with autism, 18 children with developmental delay and 18 typically developing children and found that the group with children with autism experienced weaker imitation skills. Based on the same concept, a study using Snatcher model of the LEGO mindstorm has been developed to impart psychomotor skills through imitation [29]. The LEGO Snatcher was programmed to perform 4 activities and the children were trained to imitate the same. It was found that this mode of training showed significant improvement in imitation and psychomotor skills. 'Romibo', a robot used to assist therapists, shows emotions and can forge relation- 
ships with children [30]. It may be noted that most of these robots are used for cognitive \& social skills and not for psychomotor skills [31]. Further, most of the aforementioned studies work with high cost robots, whereas this paper presents a frugal mechatronic training kit which is affordable by special schools, therapists and parents in developing countries like India.

\section{Identified task and associated skills}

In daily situations, on many occasions one needs to hold an object and rotate to get some desired results, such as opening/closing a bottle cap or a room door. Though it may appear to be a simple task for typically developing children, it is a challenge to children with autism to perform such actions. This task involves gross motor skills like elbow movement (reaching for the object) and grasping action (placing the palm on the object) and fine motor skills like palmar grasp (holding the object using all five fingers wrapped around it) and wrist rotation (turning and twisting of the object). Another important skill required is bilateral coordination that involves the meaningful use of both the hands to carry out a task. Cognitive understanding of directions such as left/right and clockwise/counterclockwise is also involved in performing certain tasks. Children with ASD lack in generalisation and find it challenging to relate the skill taught to them in one situation to another. The main aim of developing mechatronic solutions for training purpose is to enhance the concept of generalisation by training the children to perform certain tasks repetitively. Training the children by employing the proposed mechatronic door kit, to turn a knob in a certain required direction repeatedly helps them to improve their knowledge of direction and to generalise the concept of opening and closing. It also improves eye-hand coordination, which is a challenge for an autistic child.

\section{Development of mechatronic door training kit}

\subsection{Design requirements and conceptual design}

Training children with autism is a tedious process, since it calls for consistent and repetitive execution of tasks, which is a challenge for the instructors. Hence, develop- ing simple, cost effective mechatronic kits for assisting the instructors in training is the need of the hour. As mentioned in section 2, one of the major challenges faced by the children with autism in day-to-day life is to perform actions such as opening/closing of the door involving finger grasps and wrist rotations [32]. To make the training easier for both the therapist/special educator and the child, a mechatronic door training kit has been conceived. The kit consists of a miniature door hinged to a frame, which could be opened or closed by rotating two different knobs. The system needs to be portable and with minimum area of foot print. One of the requirements of the system is programmability of tasks to execute an activity several times repeatedly. The system needs to be programmed in such a way that, during the training of a specific task, e.g. 'open' operation or 'close' operation, the door closes or opens automatically after each trial. This is owing to the fact that the children would get distracted if the therapist/special educator manually closes or opens the door after each trial.

Care needs to be taken in designing the system and making it child friendly. The knobs are the only elements in the entire system that children will have a physical interaction with. Hence, the selection of the knob is very important. They have to be small to fit within a child's palm and should be spherical in shape to represent a real life doorknob. The original texture of the knob can be very smooth, but several other textured materials are to be kept as alternatives to cater to child's difficulty in touch sensory integration [33]. The location of the knobs on the system has to be determined based on the physical and visual accessibility of the child. The system is to be painted in bright colours to attract, induce positive behaviour and sustain the child's attention throughout the training $[11,34]$. An attractive object like a bright coloured doll if placed behind the door, can act as a positive reinforcement for the child's focus and concentration [35].

\subsection{Description of the kit and design details}

Figure 1 shows the training kit of dimensions $46 \mathrm{~cm}$ (L) $\times 25 \mathrm{~cm}(\mathrm{~B}) \times 24 \mathrm{~cm}(\mathrm{H})$. It consists of a wooden platform, on which a doorframe with dimensions $21 \mathrm{~cm}(\mathrm{~L}) \times 19 \mathrm{~cm}$ (H) has been erected. It has a door of $14 \mathrm{~cm}(\mathrm{~L}) \times 15 \mathrm{~cm}$ $(\mathrm{H})$ hinged to it. While the platform and the frame are made of wood, the door is made from an acrylic sheet for lightweight. Two steel knobs are placed on the wooden plank in front of the door on the right side, for easy access. The knobs are separated from each other by a distance of $2 \mathrm{~cm}$ and they are specifically placed in the field of view of the children to minimize the chances of distraction and 
loosing focus. Each knob is mounted onto a potentiometer shaft, which is projecting from underneath the wooden plank. The kit is designed to open or close the door when the knobs are rotated in a particular direction by actuating a motor through a link mechanism. The potentiometer attached to the right knob when rotated clockwise will facilitate opening of the door. Voltage varies depending on the extent of turn of the knob. At the home position of this potentiometer, the voltage measured across it is $0 \mathrm{~V}$ and when rotated, can reach a maximum of $5 \mathrm{~V}$. The potentiometer of the left knob is connected in such a way that it rotates in counter-clockwise direction. The voltage across this potentiometer is $5 \mathrm{~V}$ at home position and can reach $0 \mathrm{~V}$ by turning the knob.

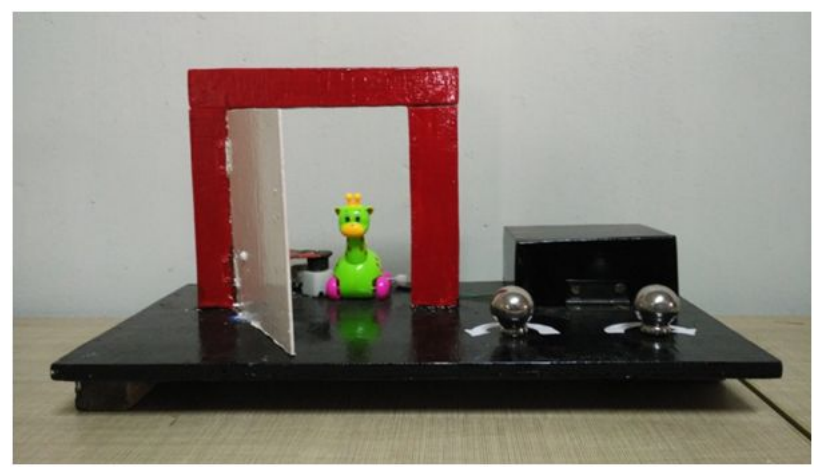

Figure 1: Mechatronic door training kit.

The software residing in the control unit is responsible for the entire operation of the kit. An Arduino UNO module acts as the control unit. When the knob is operated, the analog signal from the potentiometer is fed to the analog input pins of the board. The corresponding control signals from the output pins are fed to the DC motor through an L293D motor driver circuit. The power required to drive the motor is supplied through a voltage regulator circuit which, employs an IC 7805.

\subsection{Working of the system}

The door model of the kit has been designed to execute three different tasks 'open', 'close' and 'open \& close'. To open the door, the right knob is rotated in clockwise direction, which in turn rotates the potentiometer connected to it. When the voltage from the right knob is more than the maximum threshold value of $3.5 \mathrm{~V}$, the board generates a signal to rotate the motor in clockwise direction, thus opening the door. If the voltage is below $3.5 \mathrm{~V}$ then the kit waits until the necessary voltage is reached (until the knob is rotated to the required extent) to execute the required motion. Similarly, when the left knob is rotated in counter-clockwise direction, to give an output voltage less than minimum threshold value of $1.5 \mathrm{~V}$, the board generates signal to rotate the motor in counter-clockwise direction to close the door. The special educator should bring the knobs back to home position after each trial. The process for the task 'open \& close', is started when the right knob is rotated in the clockwise direction to open the door and ends with the rotating of the left knob in counterclockwise direction to close the door. To make the operation of the kit easier for the therapist [35] and also to reduce distraction for the children, during the 'open' task, the door closes automatically after 5 seconds of opening. Similarly, during the 'close' task, the door opens automatically after 5 seconds of closing. This is also to inculcate waiting in children with ASD and to avoid any kind of mishandling by the child immediately after the action.

\section{a) The role of therapist/special educator during training with the kit}

A few days before the start of the training involving the children, all the therapists/special educators who would be accompanying them to the therapy, were assembled together and hands on training was provided. First the concept, then the working and the need for the system was explained, following which the trial procedures were demonstrated. Later every therapist/ special educator carried out the trials one by one and acquired hands on experience on the system.

During the training with the kit, involving the children, the therapist/special educator initially demonstrates to the child how to open and close the door using the kit. Later, the selected task is physically taught to the child. After this, the first command is issued and the therapist/special educator waits for the child to respond, if the child does not respond then required assistance is to be provided by using appropriate prompts, like physical cues, touch cues or oral cues. The therapist/special educator needs to keep the children focused on the kit throughout the training session. After each trial, the therapist/special educator brings back the knobs to the home position.

\section{b) Child's part during training}

The child is made to sit in front of the kit facing it, with the special educator sitting right next. The child initially gets familiarised with the kit, through demonstration and teaching by the special educator. Later when instructed by the special educator, the child is expected to respond by holding the corresponding knob and rotate it to open or close the door as required. Once the door is opened or closed, the child needs to wait and also observe for the 
door to automatically close or open respectively. After each trial the child needs to wait until the next instruction is given by the therapist/special educator.

\section{Trials}

In order to study the usefulness of employing the mechatronic door training kit, six children with autism aged four to nine years studying in a special school in Tiruchirapalli, Tamil Nadu, India, were considered. They were undergoing speech and occupational therapies provided in the special school. All the children were found to be right - handed. The trials were conducted on a one on one basis in a closed room ensuring minimal distractions. The training sessions were performed by respective special educators of the children while the observations were recorded by one of the authors. The sessions were entirely video recorded with the signed consent of the parents, for further studies. The study consisted of three sections viz; preassessment, training and post-assessment for each child. The pre-assessment was to find out the state of the children's knowledge and skill before the training and the post-assessment to see the improvement in the children's ability after training.

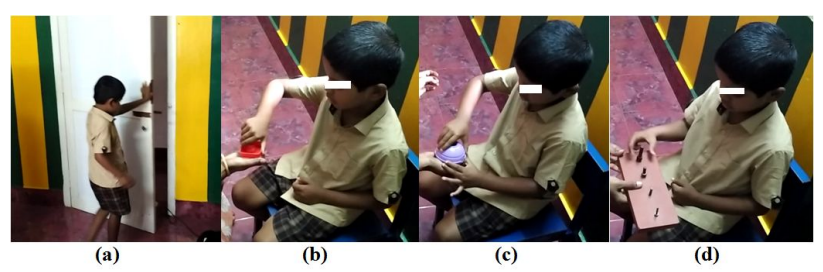

Figure 2: Snapshots of pre-assessment activities (a) Opening and closing of door; (b) Rotating a cubical toy; (c) Twisting a hemispherical toy; (d) Screwing action using bolt-nut arrangement.

\subsection{Pre-assessment}

In pre-assessment, the children were given four simple daily life activities to execute. A snapshot from the recorded video graphs, of various pre-assessment activities, of a child is shown in Figure 2. The assessment of each activity was recorded using a four-point scale, with zero corresponding to the absence of any response and four corresponding to success without assistance. The rating reduces depending upon the assistance provided to complete the task as shown in Table 1.
In the first activity, the child was made to stand in front of a real door present in that room and was instructed to 'open the door', 'close the door', 'open the door using right hand' and 'close using left hand'. The instructions were given one after the other, giving sufficient time for the child to respond without any other prompts. The child would either respond and execute it correctly or incorrectly, or would not respond. The respective outcomes were noted down. This was to basically check the child's familiarity with door, ability to open/close the door and to distinguish between right and left hands. Figure 2(a) shows a snapshot of the child performing pre-assessment activity of opening and closing the door.

The second and third activities were to rotate a cubical toy like object with two parts joined together by means of screw thread arrangement and a hollow sphere made of two hemispheres that can be twisted as shown in Figures 2(b) and 2(c). The child was instructed to turn one part of the toy while the other was held by the special educator in her palm. This again was done using both the hands successively one after the other and the outcomes were recorded respectively.

The fourth activity was with a small wooden board having different sizes of bolts and nuts as shown in Figure 2(d). The children were instructed to move the nuts using screwing action from one end of the bolt to the other using one hand at a time. These activities were to assess the ability of each child to hold an object using palmar grasp, to twist and turn using his/her wrist and distinguish between his/her left and right hands. The outcomes of the pre-assessment trials were compiled for further studies.

\subsection{Training}

\section{a) Objectives and expected outcomes of the tasks}

The process flow of the trials is explained below: Three tasks are created based on the developed mechatronic door training kit. In the first task, the child will be trained to 'open' the door. When the child turns the right knob clockwise using his/her right hand, the door opens and in 5 seconds, the door will automatically close. This task is mainly to familiarize the child with the opening of doors. The 5 seconds wait before the automatic closing of the door is to enforce the child to notice the working of the door, which is aimed to develop focus and attention. The second task is to 'close' the door. When the child turns the left knob counter-clockwise using his/her left hand the door closes and in 5 seconds, it opens automatically getting ready for the next trial. This task is mainly to familiarize the child with the closing of doors. The third task is to 
Table 1: Assignment of scores.

\begin{tabular}{lcc}
\hline \multicolumn{1}{c}{ Observations } & Markings & Score \\
\hline Absence of response & $\boldsymbol{x}$ & 0 \\
Physical cue & JTeach & 1 \\
Touch cue & JTouch & 2 \\
Oral cue & $\sqrt{ }$ OC & 3 \\
Independent \& correct response & $\checkmark$ & 4 \\
\hline
\end{tabular}

both 'open' and 'close' the door successively, thus helping children to familiarize and also to generalize the idea of door opening/closing in everyday life. When the child operates the right knob, the door opens and when it operates the left knob the door closes. The reason behind training the child to use specific hands is to help the child distinguish between left and right hands and also enhance bilateral coordination. Unambiguous hand preferences lead to good motor skills [5]. The educational objectives of the training using this kit include the following: i) to listen to command, understand and associate it with the appropriate hand, ii) to move that hand to hold the knob using 'palmar grasp' and iii) to turn the knob using 'wrist rotation' which in turn would open or close the door. Thus, the child is expected to learn to listen, remember, choose, associate and execute the task as per the given instruction.

\section{b) Trials procedure}

Subsequent to pre-assessment, each child was given training using the mechatronic door training kit. The total training for each child spanned over four sessions comprising of 'open' task in session 1, 'close' task in session 2 and combination of 'open' and 'close' tasks in sessions 3 and 4. Each training session comprised of 10 trials each.

Initially the mechatronic training kit was introduced to the child, by pointing and telling him/her what the parts were. Later the first task was demonstrated to the child by operating it. The special educator then physically taught the child by holding the child's hand over the knob and operating the knob to open the door. After the child was physically taught once, instruction to open the door using right hand was given. If the child did not respond then the required prompt was given to the child. If the child did not completely respond then the child was given physical prompt again, where the special educator held the child's hand and taught him/her physically how to open the door. If the prompt required was only 'touch' then the special educator just touched the child's hand appropriately. If the child responded but was just confused with the hand then oral prompt was given for the child to execute the instruction correctly.
The child was asked to wait for 10 seconds between trials so as to enhance acceptance and adaptability to a given situation. Similarly, in the second task, the door was kept in the open position, and 'close' instruction was given. The child was asked to operate the left knob using the left hand and close the door. After a programmed delay of 5 seconds the door would open automatically. After a wait of subsequent 10 seconds, the next trial was taken up. In the training session for the third task, when the instruction was issued the child operated the right knob to open the door and after a wait of 5 seconds, operated the left knob to close the door. There was a 20 second long wait before the next trial was taken up. In the third task, waiting duration was intentionally increased to 20 seconds as two tasks 'open' and 'close' were combined together and also to gradually increase the compliant behaviour of children in waiting. Also during this session, 20 trials were taken to observe the performance improvement.

\section{c) Observations}

The observations during the trials were noted in a trial sheet for each child. A typical trial sheet with the observations recorded during a training session for 'open' task is given in Figure 3. The rows correspond to trials while the columns correspond to the different parameters of the study. If the child used the right hand then $\checkmark$ was marked under the 'Hand' column. If the palmer grasp was correctly executed by the child then $\checkmark$ was marked in that appropriate cell of the 'Hold' column. If the child turned the knob and opened the door then $\checkmark$ was marked in the 'Open' column for that trial. After the door was opened, if the child waited for 5 seconds to close, then a $\checkmark$ was marked in the appropriate cell. Similarly, the 'Looking at Door' column was used to note if the child was capable of looking at the opening and closing of the door. After the door was closed if the child waited for a pre-assigned time of 10 seconds before the instruction of the next trial then $\checkmark$ was marked in the corresponding cell. If the child needed assistance then the $\checkmark$ was followed by the prompt needed, Teach for physical cue, Touch for touch cue and OC or oral cue. If the child did not respond then $\boldsymbol{X}$ was marked in the corresponding 
cell. Similar to the trial sheet of the open task, separate trial sheets for 'close' and 'open \& close' tasks were used.

\subsection{Post-assessment}

After the training sessions, a post-assessment was performed to observe the improvement in the children. The same set of activities that were given in the pre-assessment were again performed, ratings were provided on the same four-point scale and the outcomes were noted. The children who initially could not hold objects or rotate them were able to do it; there was an enhancement in palmar grasp and the activity of screwing a nut onto a bolt was also carried out in a better way. Wrist rotations were executed with much ease than before. All the children were using both hands and also could differentiate between 'left' and 'right'. They could open and close the door. Most importantly, all the children started to obey and respond to given instructions and commands. The outcomes of the post-assessment trials were compiled, column graphs were plotted and compared with the pre-assessment trial graphs to analyse the improvement of every child. This is discussed in detail in the next section.

\section{Results}

\subsection{Analysis of outcomes}

To analyse the outcomes of the training, learning curves were drawn for the identified tasks and associated skills, by assigning scores for different levels of performances of the children. Scores were assigned between 0 and 4 against various markings in the trial sheet as shown in Table 1 . To obtain overall weighted score for rating the performance of a particular task, weightages for the skills associated with the task were given in the order of complexity. A weightage of 0.2 for hand selection, 0.3 for holding the knob using palmer grasp and 0.5 for wrist rotation were used. The weighted score for each skill was computed by multiplying the assigned score with the corresponding weightage. The performance of a child in each trial was given a total weighted score, which was obtained by adding the weighted scores for each skill.

For analysis purpose, the trials were split into two categories namely 'learning' and 'assessment'. The first ten trials of 'open' activity, next ten trials of 'close' activity and the initial ten trials of 'open \& close' activity forming a total of thirty trials constituted the 'learning' cate- gory. The 'assessment' category consisted of the last ten trials of 'open \& close' activity. The purpose of the assessment curves drawn using these last ten trials was to observe whether the children have learnt the activities in the first 30 trials of training. In order to study the progress of learning continuously, learning curves were plotted using the total weighted scores obtained by all six children for the 'learning' category of trials. These learning curves are shown in Figure 4, in which blue curves represent opening of the door using right hand and red curves represent closing of the door using left hand.

From Figure 4(a), it can be observed that the learning curve of child 1, for 'open' activity (right hand) starts with a total weighted score of 1.3 and gradually increases to 4 towards the end of the tenth trial. During the first trial, the child was unable to perform palmar grasp. Moreover, she required assistance of the special educator in the form of physical cues for wrist rotation and hence the low score. Towards the end, she did not require any form of assistance to perform the 'open' activity. The dip in the learning curve, with a score of three each, at the seventh and the eighth trials is due to the touch assistance required for wrist rotation alone. Considering the 'close' activity (left hand), from eleventh trial to twentieth trials, the learning curve starts with a score of 1 as the child required assistance in the form of physical cues for all the skills (hand selection, palmar grasp and wrist rotation). The curve gradually rises to reach a steady score of 2.4 at the end of twentieth trial. The low scoring at the end of the twentieth trial could be attributed to the requirement of assistance in the form of touch cues to achieve both palmar grasp and wrist rotation. An important factor to be noted here is that- the child learnt to identify the left hand without assistance towards the end of the twentieth trial. The learning curves of 'open \& close' activity i.e. from trials twenty one to thirty, correspond to a combination of 'open' and 'close' activities and hence the curves of right and left hands are plotted in same $\mathrm{x}$ and $\mathrm{y}$ axes as shown in Figure 4(a). It can be seen that the child learnt all the skills using right hand at the end of the first ten trials itself and this continued throughout the training session as she scored the maximum, all through twenty one to thirty trials. But, the learning curve for the activity using the left hand starts at a score of 2.3 that is closer to the score obtained in the twentieth trial and ends at a score of 2.6 in the thirtieth trial. During the initial three trials of the activity using the left hand, the child required physical cue for wrist rotation and oral cue for hand selection, in contrary to the twentieth trial, and hence a score of 2.3. Here, assistance was required for hand selection because the child had to choose between the two hands as the activity was a combination of 'open' 


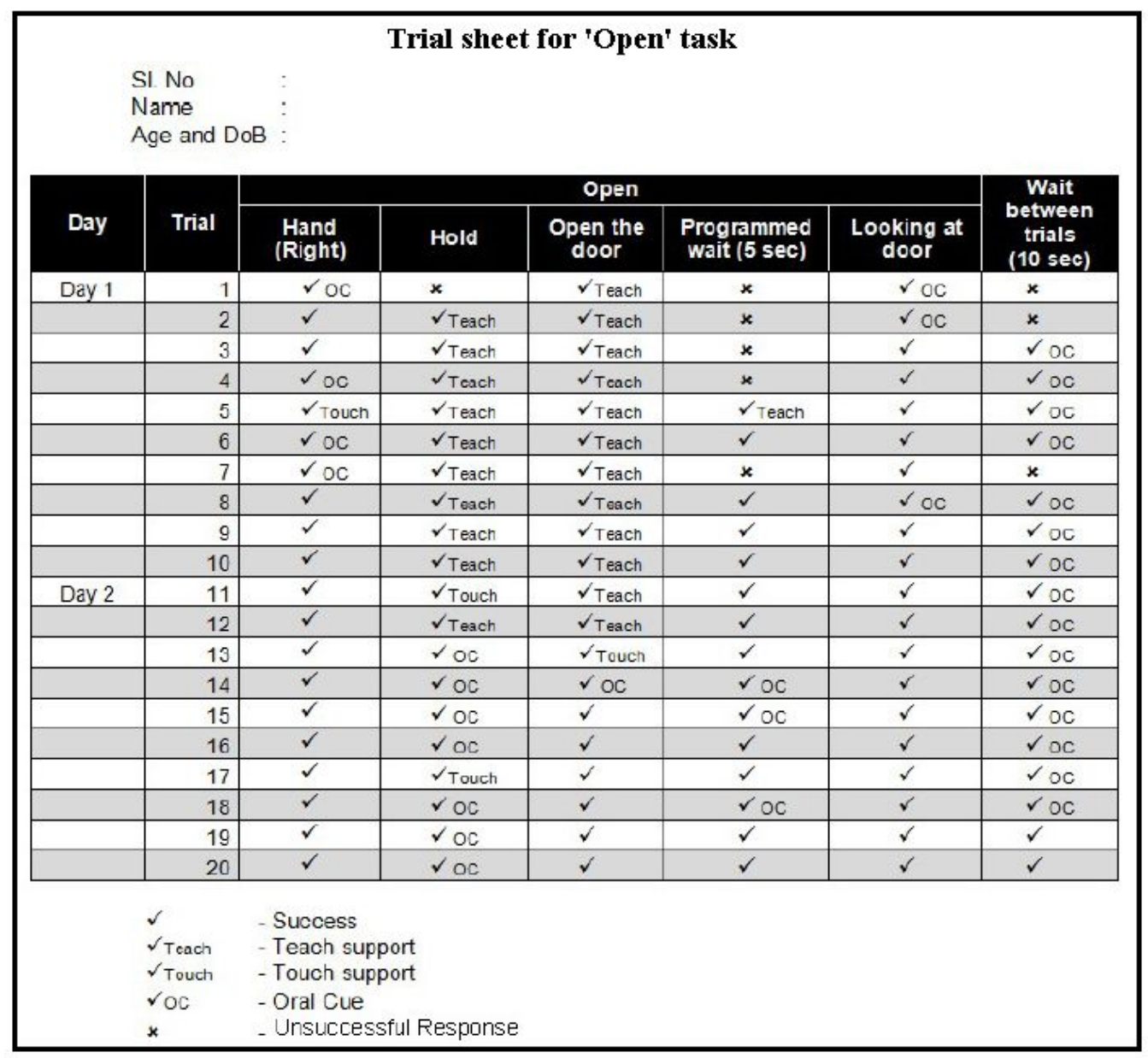

Figure 3: A typical trial sheet.

and 'close'. Towards the end of the thirtieth trial, the child learnt palmar grasp but required touch cues for hand selection and wrist rotation.

Figure 4(b) shows the learning curves of child 2 for thirty trials. The learning curve of the 'open' activity starts with a score of 2.5 where, the child needed assistance only for wrist rotation in the form of physical cue. The curve improved as the child started learning and ended at a maximum score of 4 at the end of the tenth trial. The dip in the curve at the sixth trial which corresponds to score of 2.5 is because of the physical cue that the child required to perform wrist rotation. However, gradually the curve improved thereafter. While observing the learning curve of 'close' activity, the curve started with a score of 1.9 where the child required physical cue for wrist rotation \& hand selection and the curve improved towards 3.8 at the end of twentieth trial. The child learnt palmer grasp and wrist rotation but required oral cue for hand selection. The activity using right hand at twenty first trial starts with a score of 3.6 and reached a maximum score of 4 at twenty fourth trial and scored consistently till the end. Even though the child had scored 4 during tenth trial, his score reduced to 3.6 in the twenty first trial as he required 'teach cue' for hand selection. The curve of the activity with left hand started with a score of 3.6, slightly below the score of 3.8 in twentieth trial and ended with a score of 3.8 at the end of the trials. Even though the child required minimal assistance to achieve the tasks, improvement in learning curve is visible from the first trial to the thirtieth trial.

In the learning curve of child 3 for 'open' activity, despite of several dips, the learning curve improved from a score of 1.6 to 3 as shown in Figure 4(c). The dip in the fourth trial (score-2.7) attributes towards assistance required in the form of touch cue for palmar grasp \& wrist rotation and the dips in the sixth \& seventh (score-2.1) trials attribute towards assistance required in the form of physical cue for palmar grasp and touch cue for wrist rotation. The learning curve of 'close' activity is observed to be improving gradually from the eleventh trial (score-1) to twentieth trial (score- 3.6). Both the learning curves of 'open \& 


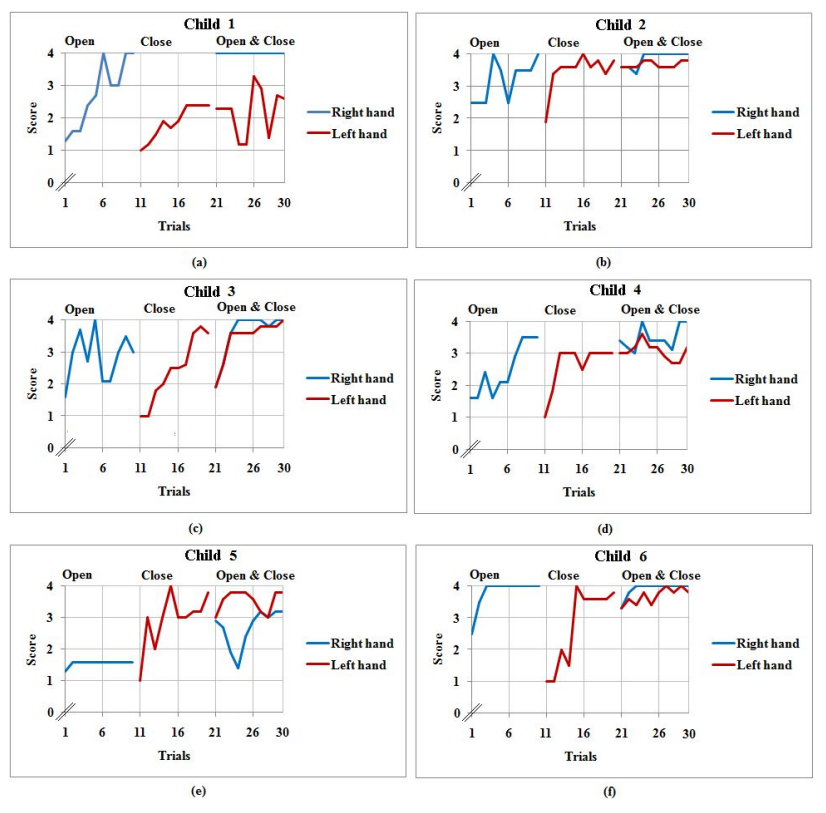

Figure 4: Learning curves of the children for the first thirty trials.

close' activity start with a score of 1.9 and gradually increase to achieve a maximum score of 4 towards the end of the thirtieth trial. An overall improvement in learning can be observed from the first trial to the thirtieth trial.

It can be observed from the learning curves of the child 4, shown in Figure 4(d), that the 'open' activity starts with a score of 1.6, improves to a score of 2.4 at the third trial but dips down back to 1.6 at the very next trial and again increases gradually to attain 3.5 towards the end. The dip in the third trial is mainly due to the assistance required in the form of physical cues for palmar grasp and wrist rotation but towards the end of this session (eighth, ninth and tenth) he learnt hand selection and palmar grasp, still needing oral cue for wrist rotation. The learning curve of the 'close' activity starts with a very low score of 1 but ends with a score of 3 starting from seventeenth trial. While the child required physical cue for achieving all the skills during the eleventh trial, his skills improved throughout the trials and required touch cues, only for hand selection \& palmar grasp. While considering the curves of 'open \& close' activity, the score of the activity with right hand starts at 3.4 and reaches 4 towards the end of the learning phase. The larger dip in twenty-eighth trial is because, the child required physical cue for palmar grasp. The score of the activity with left hand starts at 3 and ends with a score of 3.2 at the thirtieth trial as he required oral cue for hand selection and touch cue for palmar grasp.

The learning curve of child 5 depicted in Figure 4(e) shows that the child showed a consistent poor performance throughout the first ten trials of 'open' activity with a score of 1.6. He required physical cue for palmar grasp and wrist rotation throughout these trials. During the first trial of 'close' activity, the child scored only 1 as he required physical cue for all activities but at the twentieth trial he scored 3.8 as he required only oral cue for hand selection. The larger dip in the curve during thirteenth trial (score-2) is because he required assistance in the form of touch cue to achieve all the skills. Even though the learning curve of the activity with right hand, starts with a score of 2.9 and attain 3.2 in the thirtieth trial, the score dips down to a score of 1.4 during twenty fourth trial. This is because of the difficulty the child was having in performing wrist rotation and palmar grasp using right hand. The learning curve of activity using left hand starts with a score of 3 and improves to a score of 3.8 in the thirtieth trial. The dip in twenty eightieth trial (score-3) is due to the assistance required in the form of touch cue for palmar grasp and hand selection.

Child 6's learning curve for 'open' activity starts with a score of 2.5 and reaches a maximum score of 4 in third trial and remains consistent throughout the next seven trials as shown in Figure 4(f). During 'close' activity the child managed to score 1 with assistance in the form of physical cue to perform all the skills but, it can be observed from Figure 4(f) that his learning curve improved and obtained a score of 3.8 in twentieth trial. It has to be noted that he required only oral cue for hand selection. The activity with the right hand during 'open \& close' activity, started with a score of 3.3 in twenty first trial but attained a score of 4 in subsequent trials and remained consistent throughout. Similarly, the score for the activity using the left hand also started at the score of 3.3 and improved to obtain 3.8 at the end of thirtieth trial.

By studying the learning graphs of all the six children shown in Figure 4, it can be seen that, the performance of all the children improved towards the end of the trials. While the performance of the children 2, 3, 4 and 6 were good with all of them scoring 3.2 or above, child 1 and child 5 had little difficulties with their left and right hands respectively. To observe the improvement in learning, the scorings of the last ten trials of 'open \& close' activity grouped as assessment category, are used to plot assessment curves as shown in Figure 5.

From Figure 5(a) it can be observed that the child1 has carried forward the consistency in activity using right hand from the thirtieth trials as shown in Figure 4(a) throughout the trials from thirty one to forty with a score of four. The assessment curve of the activity using the left hand reached a score of 3.6 in the thirty first trial from the score of 2.6 in the thirtieth trial. The assessment curve was found to be consistent with scores between 3.6 and 3.8, 


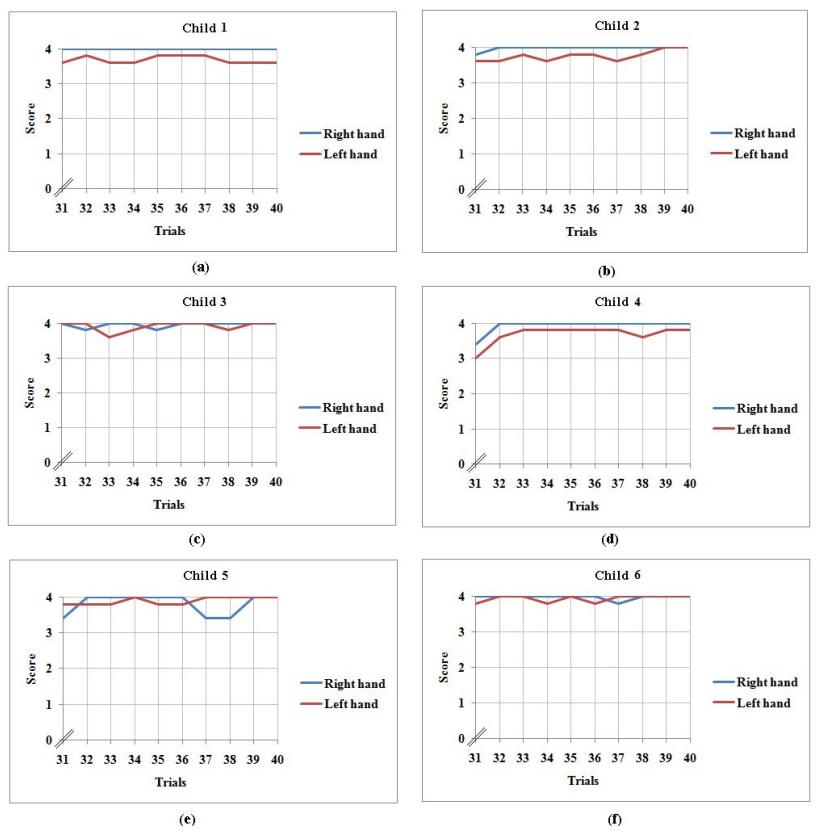

Figure 5: Assessment curves of the children for last ten trials.

with minimal assistance, until the end. The performance of child 2 for activity using right hand, depicted in Figure 5(b), shows that the score reaches the maximum at the thirty first trial and stays there until the end of the trials. Similarly, the assessment curve of the activity using the left hand starts with a score of 3.6 in the thirty first trial and improves to a score of 4 towards the end of the fortieth trial.

In the case of child 3, the assessment curves for both right and left hand activities showed almost similar scorings from thirty first to fortieth trials as shown in Figure 5(c). The assessment curve for activity using right hand for child 4 starts with a score of 3.2, achieves the maximum in the very next trial and stays at maximum until the end as shown in Figure 5(d).The curve for the activity using left hand for the same child starts with a score of 3 and improves to 3.8 towards the end of fortieth trial. Even though child 5 had difficulties in achieving 'open' activity (activity using right hand) during the first thirty trials, improvement in learning can be observed in the assessment curve of next ten trials as shown in Figure 5(e). The score of child 5 in the activity using right hand achieves the maximum by thirty second trial, dips down to 3.4 in thirty seventh and thirty eighth trials as he required minimum assistance but gained back to 4 towards the end of the trials. The child scored 3.8 during the thirty first trial of the activity using left hand and after thirty seventh trial he maintained the consistent score of 4 . The child 6 maintained almost top score with occasional requirement of minimal assistance throughout the last ten trials, for 'open\& close' activity, as shown in Figure 5(f).

\subsection{Performance improvement}

Figures 4 and 5 presented the learning and assessment curves of the six children showing the progress made in motor skills, viz. 'hand selection', 'palmar grasp' and 'wrist rotation' to accomplish the tasks of opening \& closing the door. However, in order to study the overall influence of the training in enhancing the psychomotor skills, the performance of the children during pre-assessment and post-assessment sessions have been closely compared and analysed. The performance of the children during preassessment and post-assessment sessions were recorded by assigning scores, for activities namely 'door open' using right hand and 'door close' using left hand, between 0 and 4 corresponding to the markings furnished in Table 1. For the other activities, i.e., activities using cubical toy, hemispherical toy and nut \& bolt arrangement, scores were assigned in a similar manner, individually for both right and left hands. Later the average of both was calculated to arrive at a total score for each of these activities. Using the recorded scores a column graph has been plotted as shown in Figure 6, to compare the performance of the children during pre-assessment and post-assessment. From Figure 6(a), it can be observed that the child 1 had difficulties in performing all the tasks in pre-assessment session except opening the door with right hand. It can also be observed from Figure 4(a) that the child had difficulties with palmar grasp and wrist rotation during initial trials as mentioned earlier. Later, the child learned the activities and scored well as can be seen from the assessment curves in Figure 5(a). This improvement in learning is reflected in the better performance in the post-assessment session. She was able to perform door open with right hand and door close with left hand without any assistance but required same levels of assistance to complete the remaining activities.

Child 2 could perform only activities with cubical toy and nut $\&$ bolt arrangement and that too with touch cue during pre-assessment session as shown in Figure 6(b). The learning through the training has been reflected in the post-assessment session as he was able to perform all the tasks successfully without any assistance except for a minimal assistance as oral cue for activity with hemispherical toy. Figure 6(c) shows the pre-assessment and post-assessment comparison graph of child 3 , where during pre-assessment session, the child could only perform activities using hemispherical toy and nut \& bolt arrange- 
ment. During post-assessment session, after the training, the performance of the child improved. He was able to execute the activity involving hemispherical toy without any assistance and all the other activities with some assistance in the form of oral cue.

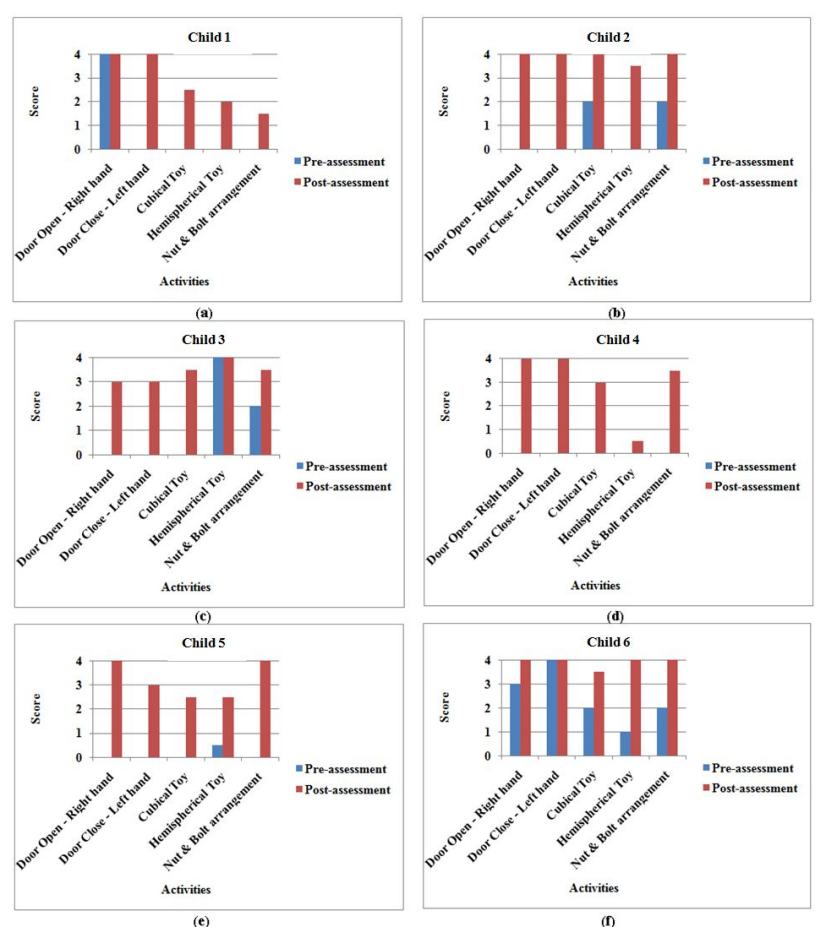

Figure 6: Pre-assessment and post-assessment comparison graphs.

In the pre-assessment session, child 4 could not perform any of the activities as can be seen from Figure 6(d) but, the performance of the child improved after the training with the kit and this is reflected in the post-assessment columns in the graph. The child required physical cue to perform the activity involving the hemispherical toy followed by assistance in the form of oral cue to perform the activity with cubical toy. To perform the activity using the nut \& bolt, he required minimal assistance and required no assistance to perform the door open and door close tasks.

It can be seen from Figure 6(e) that, child 5 could perform only the activity using hemispherical toy during pre-assessment session and that too with physical cue. It can be observed from the learning curves depicted in Figure 4(e) that the child had difficulties in using both the hands, predominantly the right hand and his performance got improved as can be seen in the assessment curves depicted in Figure 5(e). This improvement is also reflected in better performance during post-assessment session where he performed the door open with right hand and activity using nut \& bolt arrangement without any assistance and required minimal assistance to complete the other tasks.

Child 6 required some level of assistance (physical cue, touch cue) to perform all the activities in the preassessment session as shown in Figure 6(f). After training it can be seen from the graph that, there is improvement in every activity as he was able to perform almost all the activities without any assistance except the activity using cubical toy which required oral cue.

Apart from the basic skills namely, hand selection, palmar grasp and wrist rotation, the other associated skills that were developed by the training include bi-lateral coordination, eye-hand coordination and generalisation. $\mathrm{Bi}$ lateral coordination includes distinguishing between right and left hand, coordination between both the hands and also associating them to their respective knobs. Eye-hand coordination focuses on visual contact of the child with the knobs, door and doll at various stages of trials. Generalisation is another important skill because the children should be able to use the skills learnt during the training in everyday life. Though the training of opening and closing of the door was imparted using the mechatronic training kit, it has been observed from the post- assessment session that, the children were able to open and close a real time door successfully. This shows that all the children were able to generalise the skills learnt using the mechatronic kit in everyday life. There has also been improvement in bilateral coordination and eye-hand coordination as all the children performed all the other tasks also without any assistance or with minimal assistance.

\section{Conclusion}

Teaching psychomotor skills to children with autism by employing a newly developed mechatronic door training kit is found to be effective and the results are encouraging. From the analysis of training outcomes, significant developments in palmar grasp, wrist rotation and bi-lateral coordination are seen. The skills that were lacking during the pre-assessment were found to be acquired and developed by them from the training, in the post-assessment. The concept of generalization was also imparted into training in a child friendly and efficient manner, which also showed positive results in terms of response to instructions and waiting time after each task. These encouraging results motivate the authors to take the work further and develop similar mechatronic kits that impart other psychomotor skills likepincer grasp, tripod grasp, lateral pinch, depth perception, etc. in children with ASD to make 
them self-reliant and carry out their day-to-day activities. Other cognitive skills like knowledge of directions, imitation, colour identification and matching, etc. can also be addressed in future works. Further works aimed at providing technology enabled learning and management systems addressing the psychomotor skills, will pave way for enhancing the quality of life of child with ASD.

Acknowledgements: The authors are thankful to the Department of Science and Technology, Government of India, for the INSPIRE Fellowship to the first author for carrying out the research on Design and Development of Technological Solutions for the Rehabilitation of Autistic Children and also to Pravaag Transitional Centre \& School for Children with Autism, Tiruchirapalli, Tamil Nadu for facilitating the trials.

\section{References}

[1] Developmental Disabilities Monitoring Network Surveillance Year 2010 Principal Investigators; Centers for Disease Control and Prevention (CDC), Prevalence of Autism Spectrum Disorder Among Children Aged 8 Years - Autism and Developmental Disabilities Monitoring Network, 11 Sites, United States, 2010, MMWR Surveillance Summaries, 2014, 63(2),1-21

[2] J. Baio, L. Wiggins, D. L. Christensen, M. J. Maenner, J. Daniels, Z. Warren, et al., Prevalence of Autism Spectrum Disorder Among Children Aged 8 Years - Autism and Developmental Disabilities Monitoring Network, 11 Sites, United States, 2014, MMWR Surveillance Summaries, 2018, 67(6), 1-23

[3] Common Issues and Challenges facing Children on the Autism Spectrum, Research Austism, http://researchautism.net/ autism/children-and-young-people-on-the-autism-spectrum/ challenges-facing-children-on-the-autism-spectrum [Accessed: 02-Oct-2017]

[4] C. L. Hilton, Y. Zhang, M. R. Whilte, C. L. Klohr, J. Constantino, Motor impairment in sibling pairs concordant and discordant for autism spectrum disorders, Autism, 2012, 16(4), 430-41

[5] C. P. Whyatt, C. M. Craig, Motor skills in children aged 7-10 years, Diagnosed with autism spectrum disorder, Journal of Autism and Developmental Disorders, 2012, 42(9), 1799-1809

[6] M. A. Dziuk, J. C. G. Larson, A. Apostu, E. M. Mahone, M. B. Denckla, S. H. Mostofsky, Dyspraxia in autism: Association with motor, social, and communicative deficits, Developmental Medicine \& Child Neurology, 2007, 49(10), 734-739

[7] D. Green, T. Charman, A. Pickles, S. Chandler, T. Loucas, E. Simonoff, G. Baird, Impairment in movement skills of children with autistic spectrum disorders, Developmental Medicine \& Child Neurology, 2009, 51(4), 311-316

[8] M. E. Dallas, Researchers Pinpoint Brain Region that Manages Multistep Tasks, MedicineNet, 2015, http://www.medicinenet. com/script/main/art.asp?articlekey $=190783$ [Accessed: 02 Oct 2017]

[9] S. H. Fatemi, K. A. Aldinger, P. Ashwood, M. L. Bauman, C. D. Blaha, G. J. Blatt et al., Consensus paper: Pathological role of the cerebellum in autism, The Cerebellum, 2012, 11(3), 777-807

[10] A. Crippa, S. Forti, P. Perego, M. Molteni, Eye-hand coordination in children with high functioning autism and Asperger's disorder using a gap-overlap paradigm, Journal of Autism and Developmental Disorders, 2013, 43(4), 841-850

[11] J. A. Hauck, D. Dewey, Hand preference and motor functioning in children with autism, Journal of Autism and Developmental Disorders, 2001, 31(3), 265-277

[12] S. E. Gutstein, H. R. Gutstein, The RDI book: forging new pathways for autism, Asperger's and PDD with the Relationship Development Intervention program, Connections Center Pub, 2009

[13] B. Miller, Autism affects motor skills, study indicates, Medical Xpress., 2012, https://source.wustl.edu/2012/02/autismaffects-motor-skills-study-indicates/ [Accessed: 02-0ct-2017]

[14] A. N. Bhat, R. J. Landa, J. C. (Cole) Galloway, Current perspectives on motor functioning in infants, children, and adults with autism spectrum disorders, Physical Therapy, 2011, 91(7), 11161129

[15] T. Charman, J. Swettenham, S. Baron-Cohen, A. Cox, G. Baird, A. Drew, Infants with autism: An investigation of empathy, pretend play, joint attention, and imitation, Developmental Psychology, 1997, 33(5), 781-789

[16] P. Teitelbaum, O. Teitelbaum, J. Nye, J. Fryman, R. G. Maurer, Movement analysis in infancy may be useful for early diagnosis of autism, Proceedings of the National Academy of Sciences, 1998, 95(23), 13982-13987

[17] E. Bremer, R. Balogh, M. Lloyd, Effectiveness of a fundamental motor skill intervention for 4-year-old children with autism spectrum disorder: A pilot study, Autism, 2015, 19(8), 980-991

[18] E. Jasmin, M. Couture, P. McKinley, G. Reid, E. Fombonne, E. Gisel, Sensori-motor and daily living skills of preschool children with autism spectrum disorders, Journal of Autism and Developmental Disorders, 2009, 39(2), 231-241

[19] Types of Autism Interventions, Treatments and Therapies, Research Autism, 2015, http://researchautism.net/autisminterventions/types/motor-sensory-interventionsv [Accessed: 02-Oct-2017]

[20] J. J. Cabibihan, H. Javed, M. Ang, S. M. Aljunied, Why robots? A survey on the roles and benefits of social robots in the therapy of children with autism, International Journal of Social Robotics, 2013, 5(4), 593-618

[21] B. Scassellati, How social robots will help us to diagnose, treat, and understand autism, Robotics Research, 2007, 552-563

[22] J. Lee, G. Obinata, D. Stefanov, C. Nagai, A preliminary study on the response of autistic persons to a robot feedback based on visual stimuli, Paladyn, Journal of Behavioral Robotics, 2013, 4(2), 73-82

[23] T. Goldsmith, L. LeBlanc, Use of technology in interventions for children with autism, Journal of Early and Intensive Behavior Intervention, 2004, 1(2), 166-178

[24] A. Billard, Robota: Clever toy and educational tool, Robotics and Autonomous Systems, 2003, 42(3-4), 259-269

[25] G. Kronreif, Robot systems for play in education and therapy of disabled children, Studies in Computational Intelligence, 2009, 243, 221-234

[26] I. Ranatunga, N. A. Torres, R. Patterson, N. Bugnariu, M. Stevenson, D. O. Popa, RoDiCA: A human-robot interaction system for treatment of childhood autism spectrum disorders, Proceedings of 5th International Conference on PErvasive Technologies 
Related to Assistive Environments (PETRA'12), Heraklion, Crete, Greece, 2012

[27] R. S. Moorthy, S. Pugazhenthi, Teaching Psychomotor skills to autistic children by employing a robotic training kit: A pilot study, International Journal of Social Robotics, 2017, 9(1), 97108

[28] W. L. Stone, O. Y. Ousley, C. D. Littleford, Motor imitation in young children with autism: What's the object?, Journal of Abnormal Child Psychology, 1997, 25(6), 475-485

[29] R. S. Moorthy, S. Pugazhenthi, Imitation based training to enhance psychomotor skills in autistic children using a snatcher robot, Proceedings of 2016 International Conference on Robotics and Automation for Humanitarian Applications (RAHA), Kollam, India, 2016

[30] A. Shick, Romibo robot project - An open-source effort to develop a low-cost sensory adaptable robot for special needs therapy and education, Proceedings of ACM SIGGRAPH 2013 Studio Talks (SIGGRAPH'13), Anaheim, California, 2013
[31] J. J. Diehl, L. M. Schmitt, M. Villano, C. R. Crowell, The clinical use of robots for individuals with autism spectrum disorders: A critical review, Research in Autism Spectrum Disorders, 2012, 6(1), 249-262

[32] F. J. David, G. T. Baranek, C. Wiesen, A. F. Miao, D. E. Thorpe, Coordination of precision grip in 2-6 years-old children with autism spectrum disorders compared to children developing typically and children with developmental disabilities, Frontiers in Integrative Neuroscience, 2012, 6, 1-13

[33] A. J. Ayres, L. S. Tickle, Hyper-responsivity to touch and vestibular stimuli as a predictor of positive response to sensory integration procedures by autistic children, American Journal of Occupational Therapy, 1980, 34(6), 375-381

[34] J. Lee, H. Takehashi, C. Nagai, G. Obinata, D. Stefanov, Enhancement of the communication effectiveness of interactive robots for autism therapy by using touch and colour feedback, Paladyn, Journal of Behavioral Robotics, 2014, 5(1), 53-63

[35] P. G. Esteban, P. Baxter, T. Belpaeme, E. Billing, H. Cai, H.-L. Cao et al., How to build a supervised autonomous system for robotenhanced therapy for children with autism spectrum disorder, Paladyn, Journal of Behavioral Robotics, 2017, 8(1), 18-38 